\title{
Reorganización del Conocimiento Matemático en la Elaboración de Simuladores con GeoGebra: Análisis de una actividad que envuelve la noción de Cuadrado
}

\author{
Stephanie Díaz-Urdaneta ${ }^{1}$ \\ stephaniediazurdaneta@gmail.com \\ https://orcid.org/0000-0002-8335-2022 \\ Luzia Regis Narok Pereira ${ }^{2}$ \\ matematicaluzia@gmail.com \\ https://orcid.org/0000-0001-8423-7934 \\ ${ }^{1}$ Asosiación Aprender en Red (Venezuela) \\ ${ }^{2}$ Colégio Estadual Santos Dumont (Brasil)
}

Recibido: 31/05/2020 Aceptado: 03/08/2020

\begin{abstract}
Resumen
El uso de tecnologías digitales en contextos educativos ha favorecido las posibilidades de visualización y experimentación en la Educación Matemática, lo que ha permitido una reorganización del conocimiento matemático en el desarrollo de la actividad escolar realizada. En este sentido, en este trabajo se analiza una experiencia en la cual, desde la visualización y experimentación mediada por tecnologías digitales, un alumno de Educación Media de Venezuela reorganiza sus ideas sobre lo que es un cuadrado, en la actividad de Elaboración de Simuladores con GeoGebra (ESG). Para ello, se comenzó contextualizando la investigación realizada, continuando con lo que es ESG y, posteriormente, cómo esta se entiende según la perspectiva de lo que es la reorganización mediada por las tecnologías digitales y las ideas de los Seres-humanos-con-medios. La metodología de este trabajo es cualitativa, de naturaleza descriptiva/interpretativa, en el cual se presentó el contexto, los participantes, la fuente de los datos y su análisis. Entre los resultados, en el discurso de los participantes, se observa la reorganización de lo que es un cuadrado a partir de la visualización y experimentación de las características de esta figura en GeoGebra. A partir de lo analizado, se considera que los docentes pueden tener iniciativas para llevar a cabo actividades mediadas por tecnologías digitales que ayuden en la reorganización de los conceptos geométricos.
\end{abstract}

Palabras clave: Reorganización; Visualización; Experimentación; Elaboración de Simuladores; GeoGebra.

\section{Reorganização do Conhecimento Matemático na Elaboração de Simuladores com o GeoGebra. Análises de uma atividade que envolve a noção de Quadrado}

\section{Resumo}

O uso das tecnologias digitais nos contextos educativos tem favorecido as possibilidades de visualização e experimentação na Educação Matemática, o que tem permitido uma reorganização do conhecimento matemático no desenvolvimento da atividade escolar realizada. Neste sentido, neste trabalho analisa-se uma experiência na qual a partir da 
visualização e experimentação mediadas pelas tecnologias digitais, um aluno da Educação Média da Venezuela reorganiza suas ideias sobre o que é um quadrado, na atividade de Elaboração de Simuladores com o GeoGebra (ESG). Para isso, iniciou-se contextualizando da pesquisa realizada, continuando com o que é a ESG e, posteriormente, como ela é entendida segundo as perspectiva do que é a reorganização mediada pelas tecnologias digitais e das ideias de Seres-humanos-com-mídias. A metodologia deste trabalho é qualitativa, de natureza descritiva-interpretativa, sendo apresentado o contexto, participantes, a fonte dos dados e a análise dos mesmos. Dentre os resultados, no discurso dos participantes, percebe-se a reorganização do que é um quadrado a partir da visualização e experimentação das características desta figura no GeoGebra. A partir do analisado, considera-se que os professores podem ter iniciativas para realizar atividades mediadas por tecnologias digitais que ajudem na reorganização dos conceitos geométricos.

Palavras-chave: Reorganização; Visualização; Experimentação; Elaboração de Simuladores; GeoGebra.

\title{
Reorganization of Mathematical Knowledge in the Elaboration of Simulators with GeoGebra. Analysis of an activity that involves the notion of Square
}

\begin{abstract}
The use of digital technologies in educational contexts has favored the possibilities of visualization and experimentation in Mathematical Education, which has allowed a reorganization of mathematical knowledge in the development of the school activity carried out. In this sense, this work analyzes an experience in which a student from Venezuela's middle school reorganizes his ideas about what a square is, through Simulator Development with GeoGebra (SDG). For this, the research was contextualized, and then continued defining what SDG is and how it is understood according to the perspective of what the reorganization mediated by digital technologies is and the ideas of Human-beings-with-media. The methodology of this work is qualitative, of a descriptive-interpretative nature, being presented the context, participants, the source of the data and their analysis. Among the results, in the speech of the participants, it is observed the reorganization of what a square is, starting from the visualization and experimentation of the characteristics of this figure in GeoGebra. From the analyzed information, it is considered that teachers can have initiatives to carry out activities mediated by digital technologies that help in the reorganization of geometric concepts.
\end{abstract}

Keywords: Reorganization; Visualization; Experimentation; Elaboration of Simulators; GeoGebra.

\section{Introdução}

Durante os anos 2013 e 2017, a Associação Civil Aprender en Red promoveu, em algumas instituições escolares públicas do Estado Zulia (Venezuela), a atividade de Elaboração de Simuladores com o GeoGebra (ESG). Nessa atividade, professores de 
Matemática, futuros professores de matemática e alunos estudantes da educação média (11 a 17 anos), dedicavam-se a elaborar simuladores de fenômenos artificiais ou naturais, a partir de modelos geométricos, utilizando as diferentes ferramentas e funcionalidades do Software de Matemática Dinâmica GeoGebra (Prieto \& Gutiérrez, 2015, 2016, 2017). Nesta atividade, uma das questões que foram discutidas de forma empírica foi quanto às possibilidades de reorganização do conhecimento matemático por parte dos alunos que participavam da atividade. Porém, considera-se necessário que essas experiências possam ser submetidas a uma análise qualitativa para compreender se, de fato, se teve alguma reorganização do conhecimento.

Sobre a reorganização, pode-se afirmar que o uso das tecnologias digitais, tem gerado uma forma diferenciada no desenvolvimento de qualquer atividade humana. Essa questão tem gerado uma reorganização do pensamento humano e, por consequência, a reorganização da atividade humana (Tikhomirov, 1998). No âmbito escolar, a influência da tecnologia tem gerado mudanças nas atividades desenvolvidas em sala de aula. Este assunto foi apresentado por diversos autores que comentam sobre a reorganização do conhecimento matemático no contexto educativo (Borba, 2007; Villa \& Borba, 2011; Villa \& Ruiz, 2010; Villa, et al, 2013). Em tais estudos, o uso das tecnologias digitais é assumido como elemento que tem favorecido a aprendizagem dos alunos, influenciando no surgimento de novas formas de produção do conhecimento (Villarreal, 2012). Esta questão traz a reflexão sobre o que essas novas formas de aprender na atividade Matemática produzem de novas experiências para os envolvidos no desenvolvimento de tal atividade.

$\mathrm{Na}$ Educação Matemática, uma das questões que têm sido reorganizadas com o surgimento das tecnologias digitais é a possibilidade de visualização e experimentação. Segundo Borba e Villarreal (2005), embora não representem total formalidade para demonstrações Matemáticas, na Educação Matemática representam oportunidades favoráveis para a produção do conhecimento. Para esses autores, a visualização e a experimentação neste contexto já existia, mas essas possibilidades também foram reorganizadas pela influência das tecnologias. A importância desta questão é que a reorganização da visualização e experimentação na Educação Matemática pode permitir também a reorganização do conhecimento matemático que está sendo produzido (Borba \& Villarreal, 2005). Mas é 
importante desenvolver estudos que permitam ter informações ao respeito para sua compreensão (Villa, et al, 2013).

Assim, a partir da discussão introduzida, de forma empírica, este trabalho analisa, por meio de uma experiência de ESG, a reorganização do conhecimento matemático de um aluno a partir da visualização e experimentação possível com o GeoGebra, de modo que, o objetivo foi elucidar e analisar possíveis contribuições da ESG para a reorganização do conhecimento acerca de um objeto matemático. E, no âmbito da área de pesquisa, contribuir e incentivar aos professores para o desenvolvimento de atividades com a utilização das tecnologias digitais que permitam aos alunos ter experiências de visualização e experimentação como uma oportunidade para a reorganização do conhecimento matemático escolar.

\section{A Elaboração de Simuladores com o GeoGebra}

A Elaboração de Simuladores com o GeoGebra (ESG) foi uma atividade promovida pela Associação Civil Aprender en Red. Especificamente, desenvolvida pelo Grupo TEM: Tecnologías en la Educación Matemática, uma comissão pertencente à associação. Essa atividade aconteceu nos Clubes GeoGebra, espaços educativos não convencionais consolidados em algumas instituições escolares públicas do Estado Zulia, na Venezuela. Esses clubes funcionaram entre os anos 2013 e 2017 no mesmo estado, os quais eram formados por alunos da Educação Média Venezuelana, cujas idades estavam entre os 11 e 17 anos, e por professores ou futuros professores de Matemática, estudantes da Universidade do Zulia.

A ESG era uma atividade na qual o foco principal era representar um fenômeno, natural ou científico, através de modelos geométricos (representações gráficas de expressões algébricas eram utilizadas em algumas situações) eram construídos na interface gráfica do GeoGebra, a partir das diferentes ferramentas, funcionalidades e opções dinâmicas que o software oferece (Prieto \& Gutierrez, 2015, 2016, 2017). Tais modelos representam os simuladores elaborados e são conhecidos como simuladores computacionais pelo fato de serem produzidos na interface de um software. Alguns exemplos deste tipo de simuladores podem ser encontrados em Prieto e Gutiérrez (2015, 2016, 2017), nos quais se apresentam experiências de ESG. 
A elaboração desta classe de simuladores é realizada a partir da resolução de uma série de tarefas de simulação que são resolvidas progressivamente (Rubio, Prieto \& Ortiz, 2016). Cada tarefa de simulação é associada a algumas das partes que compõem o fenômeno, de acordo com as informações encontradas sobre o mesmo. Neste sentido, os alunos resolviam tantas tarefas de simulação quantas partes do fenômeno visualizavam e escolhiam para representá-las.

A resolução dessas tarefas envolve a construção de desenhos dinâmicos de cada parte do fenômeno. Tais desenhos são modelos geométricos elaborados em ambientes dinâmicos que conservam suas propriedades espaciais declaradas em sua construção quando são arrastados por algum de seus elementos livres (Laborde, 1997). Tal construção do desenho era organizada nas seguintes ações: (i) realizar um esboço da parte do fenômeno para simular, com o intuito de ter uma melhor apreciação das formas que compõem o desenho (Figura 1a); (ii) interpretar as formas do esboço em termos geométricos, sinalizando os objetos geométricos que eram identificados (Figura 1b) e (iii) representar esses objetos geométricos na interface do GeoGebra (Figura 1c).

Figura 1: Exemplo das ações seguidas na construção de um desenho dinâmico

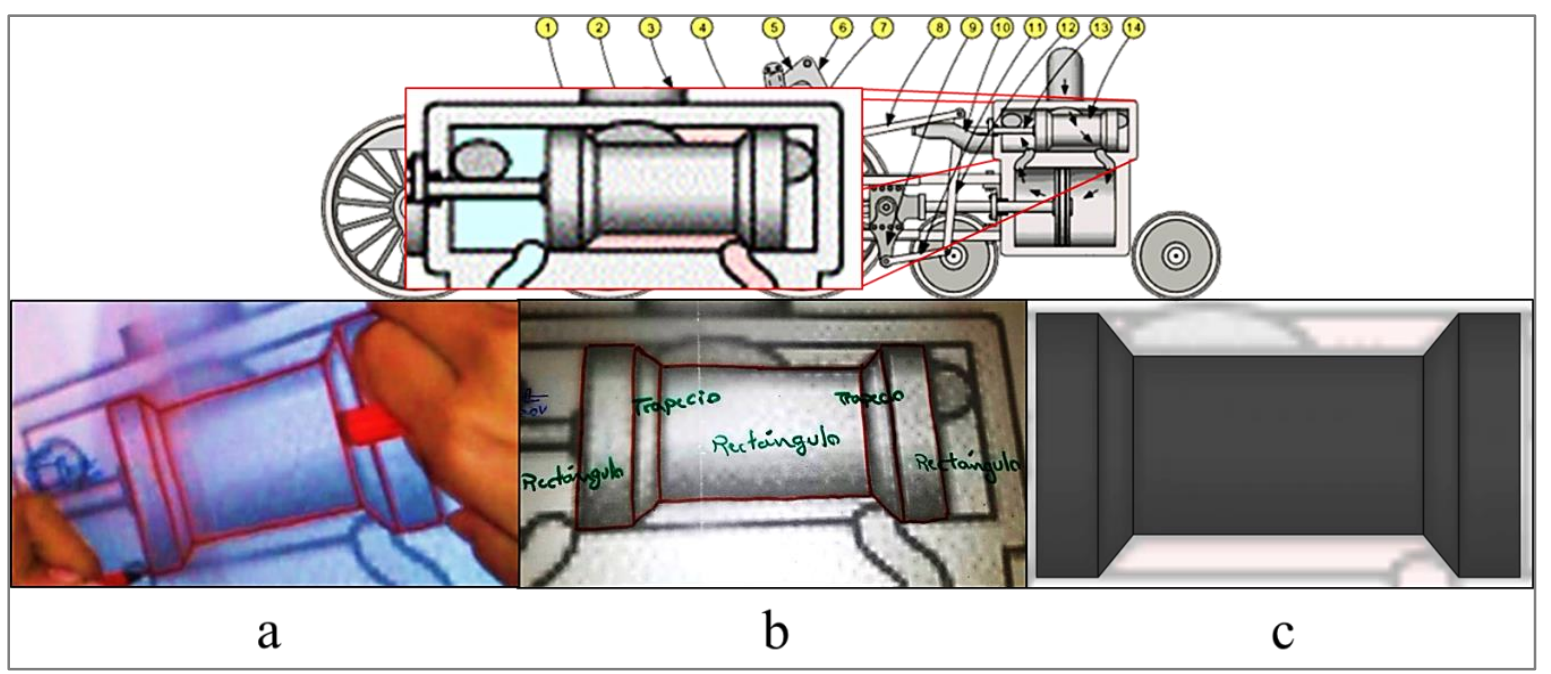

Fonte: Elaborado pelas autoras (2020)

$\mathrm{Na}$ interpretação das formas do esboço em termos geométricos, os sujeitos tentam escolher as figuras que, segundo seu olhar, pode representar com maior fidelidade à parte que está querendo ser simulada. Nessas escolhas, o uso do GeoGebra pode ser utilizado já que permite validar ou refutar se essa seleção é a mais próxima ao que se deseja representar. Esse 
momento do uso do software para tal escolha pode permitir a reorganização de alguma ideia matemática a partir da visualização e experimentação possível com o GeoGebra, quando se valida a correspondência entre a forma do fenômeno no esboço e os objetos geométricos identificados na interpretação em termos geométricos de tal esboço. Essa questão pode ser percebida nos discursos dos alunos com seus professores quando começam a transitar pelas ações mencionadas para resolver uma tarefa de simulação, revelando as ideias que os envolvidos têm. Para uma melhor compreensão destas ideias, na sequência descreve-se como o conhecimento matemático pode ser reorganizado na ESG a partir da visualização e experimentação possível no software.

\section{Reorganização do Conhecimento Matemático na ESG}

Desde a perspectiva psicológica, as relações entre as tecnologias digitais e o pensamento humano foram estudadas por Tikhomirov (1981), relações que também influenciam no desenvolvimento da atividade humana (Tikhomirov, 1981). Esse autor fez um estudo sobre três tipos de relações que podem existir entre o computador e o pensamento humano, sendo as de: substituição, suplementação e reorganização.

Sobre a relação de substituição, o autor apresenta que a atividade intelectual do ser humano pode ser substituída pelo computador, mas para Tikhomirov (1981) isso não é possível, já que o pensamento humano pode desenvolver estratégias que o computador não conseguiria. Quanto à suplementação, o computador é visto como uma ferramenta externa do pensamento humano, que aumenta consideravelmente o volume e a velocidade do processamento de informações, mas essa perspectiva também limita as capacidades do humano (Tikhomirov, 1981). Com respeito à relação de reorganização entre o computador e o pensamento, nesse contexto considera-se que a atividade humana é transformada já que influência na forma do agir do ser humano (Tikhomirov, 1981). Essa forma de relacionamento entre o homem e o computador supera essa perspectiva das tecnologias digitais como simples complementos, já que as utilizando é possível ter uma nova atividade humana que tem sido reorganizada por tal influência (Villarreal, 2000, 2003, 2004, 2013).

A partir dessa perspectiva, na Educação Matemáticas as atividades nas quais se têm possibilidades de visualização e experimentação têm mudado também. Para Borba e Villarreal 
(2005), essas possibilidades já existiam, mas têm sido reorganizadas pela influência das tecnologias digitais, portanto, também se tem uma reorganização do conhecimento matemático adquirido por pelo uso das tecnologias. Neste sentido, considera-se que a visualização e experimentação têm um papel importante nesse novo conhecimento, já que são processos com os quais é possível a representação, generalização, transformação, reflexão e comunicação sobre as informações que estão sendo visualizadas e exploradas (Hershkowitz et al., 1990; Torregrosa, 2002).

As ideias de Borba e Villarreal (2005) estão encaixadas na sua perspectiva teórica sobre Seres-humanos-com-mídias. Esse viés teórico se baseia em duas ideias centrais, sendo que a cognição é de natureza coletiva e essa mesma cognição é influenciada pelas mídias com as quais o conhecimento é produzido.

A partir destas questões, considera-se que a atividade de ESG pode ser analisada sob esses parâmetros, uma vez que o desenvolvimento da mesma é de natureza coletiva, portanto a cognição também tem essa natureza, já que envolvia, pelo menos, o aluno e ao professor ou futuro professor de matemática e está marcado pela influência do software GeoGebra como tecnologia digital com a qual é possível elaborar os simuladores.

Sobre as ideias de visualização e experimentação, neste trabalho são entendidos como processos que podem acontecer ao mesmo tempo e com os quais é possível representar, generalizar, transformar, refletir e comunicar às ideias que estejam acontecendo durante a ESG. Optou-se por esses processos já que se considera que o uso das tecnologias digitais nas experiências educacionais, permite realizar processos cognitivos diferenciados que condicionam o tipo de conhecimento matemático produzido com uma tecnologia diferente (Torregrosa, 2002; Borba \& Villarreal, 2005). Neste sentido, a reorganização do conhecimento matemático é percebida quando essas possibilidades sejam manifestadas na atividade de ESG.

\section{Metodologia}

A metodologia utilizada neste trabalho é de natureza qualitativa. Segundo Bicudo (2012) neste tipo de pesquisa o sujeito é considerado como parte do processo, sendo ele só ou com outros seres e materiais que formam parte do contexto. Tratando-se desta metodologia, Bicudo (2005, 2012) apresenta duas características: i) o objeto de estudo está colocado em um contexto, 
e ii) é possível fazer compreensões e interpretações sobre ele. Neste sentido, nem sempre é possível generalizar ou transferir as informações a outros contextos, mas oferece oportunidades para caracterizar, compreender e interpretar o que está sendo estudado. Por estas razões, a apresentação da metodologia deste trabalho realiza-se destacando o Contexto e participantes da pesquisa, os Dados da pesquisa e a Análise dos dados.

\section{Contexto e participantes}

O contexto da pesquisa foi desenvolvido em uma atividade de ESG, especificamente em um Clube GeoGebra que funcionava nas instalações de um colégio público da cidade de Cabimas, do Estado Zulia (Venezuela). Nesse clube participavam dois alunos da Educação Média Venezuelana, uma professora de matemática e um futuro professor, os quais orientavam o que acontecia no clube. Neste eram realizados sessões de trabalhos na qual eram discutidas questões próprias da elaboração de simuladores, os encontros aconteciam duas vezes por semana. Para esta pesquisa, foi selecionada uma das sessões, particularmente, uma na qual participaram o aluno Kevin (pseudônimo) e o futuro professor Rodrigo (pseudônimo). Nessa sessão discutiram sobre a identificação dos objetos geométricos sobre o esboço de uma parte de um motor de quatro tempos, cujo nome dessa parte era o contrapeso (Figura 2).

Figura 2: Esboço sobre o contrapeso do motor de quatro tempos

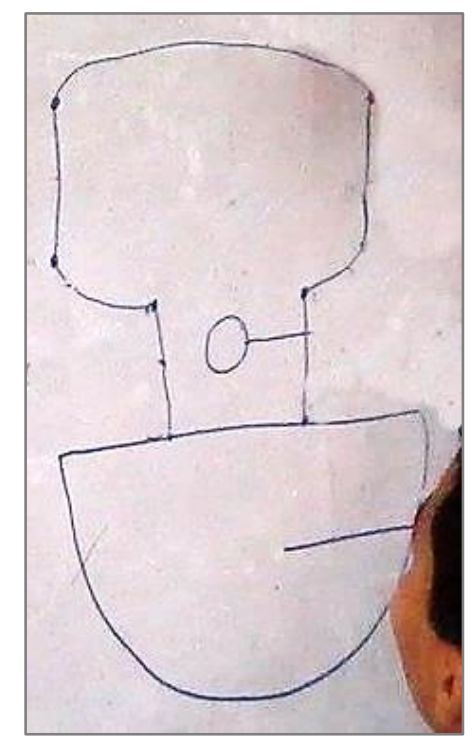

Fonte: Elaborado pelas autoras (2020) 


\section{Dados da pesquisa}

$\mathrm{Na}$ pesquisa qualitativa, alguns autores propõem que os dados sejam produzidos em vez de serem coletados, isso porque os dados, além de serem codificados pelo pesquisador utilizando os diferentes instrumentos, ferramentas e procedimentos estabelecidos, o pesquisador também influencia os mesmos, organizando-os segundo suas perspectivas (Powell \& Silva, 2015). Neste sentido, os dados da pesquisa foram produzidos a partir da filmagem em vídeo de uma sessão de trabalho dos participantes na qual eram discutidas as condições iniciais para elaboração de um desenho dinâmico para representar o contrapeso do motor de quatro tempos. Com a filmagem em vídeo foi possível capturar a realidade e os acontecimentos durante o desenvolvimento da sessão de trabalho. Os discursos realizados na sessão e registrados em vídeo foram transcritos em um processador de texto e organizados no Quadro 1, mantendo o discurso escutado, o que representa a fonte de dados da pesquisa.

Quadro 1: Instrumento para organizar o discurso da sessão analisada

\begin{tabular}{|c|c|c|}
\hline Linha & Conteúdo da transcrição & Comentários interpretativos \\
\hline 1 & & \\
\hline 2 & & \\
\hline 3 & & \\
\hline
\end{tabular}

Fonte: Elaborado pelas autoras (2020)

$\mathrm{Na}$ coluna sobre "Comentários interpretativos" foram colocadas interpretações por parte dos pesquisadores em relação às evidencias registradas nesse instrumento. Na sequencia será comentado a forma em que foi realizada a análise.

\section{Análise dos dados}

A análise dos dados foi realizada em três momentos. No primeiro momento foi realizada a leitura dos discursos que foram registrados no Quadro 1, para identificar as condições nas quais estava a elaboração do desenho dinâmico que representaria o contrapeso do motor a quatro tempo. O identificado dessa leitura ilustra-se na Figura 3. 
Figura 3: Condições iniciais para representar o contrapeso

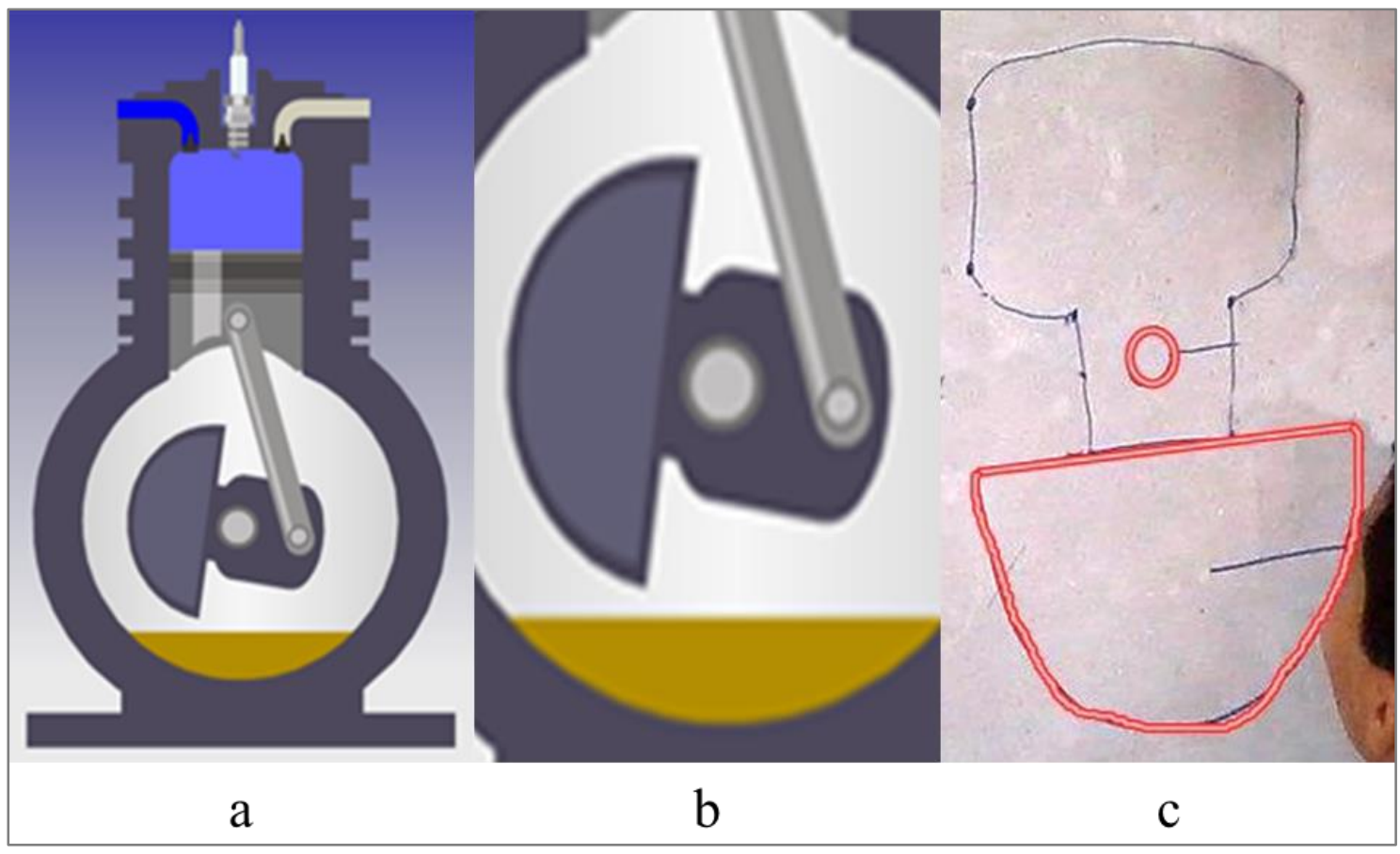

Fonte: Elaborado pelas autoras (2020)

Nessa sessão de trabalho, os participantes já haviam discutido sobre algumas questões para fazer esse desenho dinâmico que representasse essa parte do fenômeno escolhido. Primero, já tinham escolhido a imagem tomada por referência para elaborar o simulador (Figura 3a); Segundo, haviam identificado a primeira tarefa de simulação a resolver que consistia na representação do contrapeso por um desenho dinâmico (Figura 3b); e, por último, já tinham sido identificadas duas figuras geométricas sobre o esboço, um semicírculo e um círculo (Figura 3c).

No segundo momento foram identificados aqueles discursos nos quais as discussões entre os participantes deram indícios de reorganização, visualização e/ou experimentação, já que representam esses momentos de interesse para a pesquisa. No terceiro momento realizouse uma análise de conteúdo verbal que permite realizar inferências em relação às mensagens produzidas, com o intuito de destacar aquelas regularidades de interesse para a pesquisa (Bardin, 1977). Sobre esses dois últimos momentos, eles foram realizados de forma independente por cada pesquisadora, cujos resultados foram discutidos em reuniões posteriores. Na sequência estão apresentados os resultados da análise. 


\section{Resultados e Discussões}

$\mathrm{Na}$ análise dos dados foi possível reconhecer o momento em que os participantes interpretavam o esboço do contrapeso em termos geométricos, do qual já tinham identificados dois (Figura 4a) e estava faltando especificamente uma parte (Figura 4b).

Figura 4: Objetos geométricos identificados e não identificado no esboço

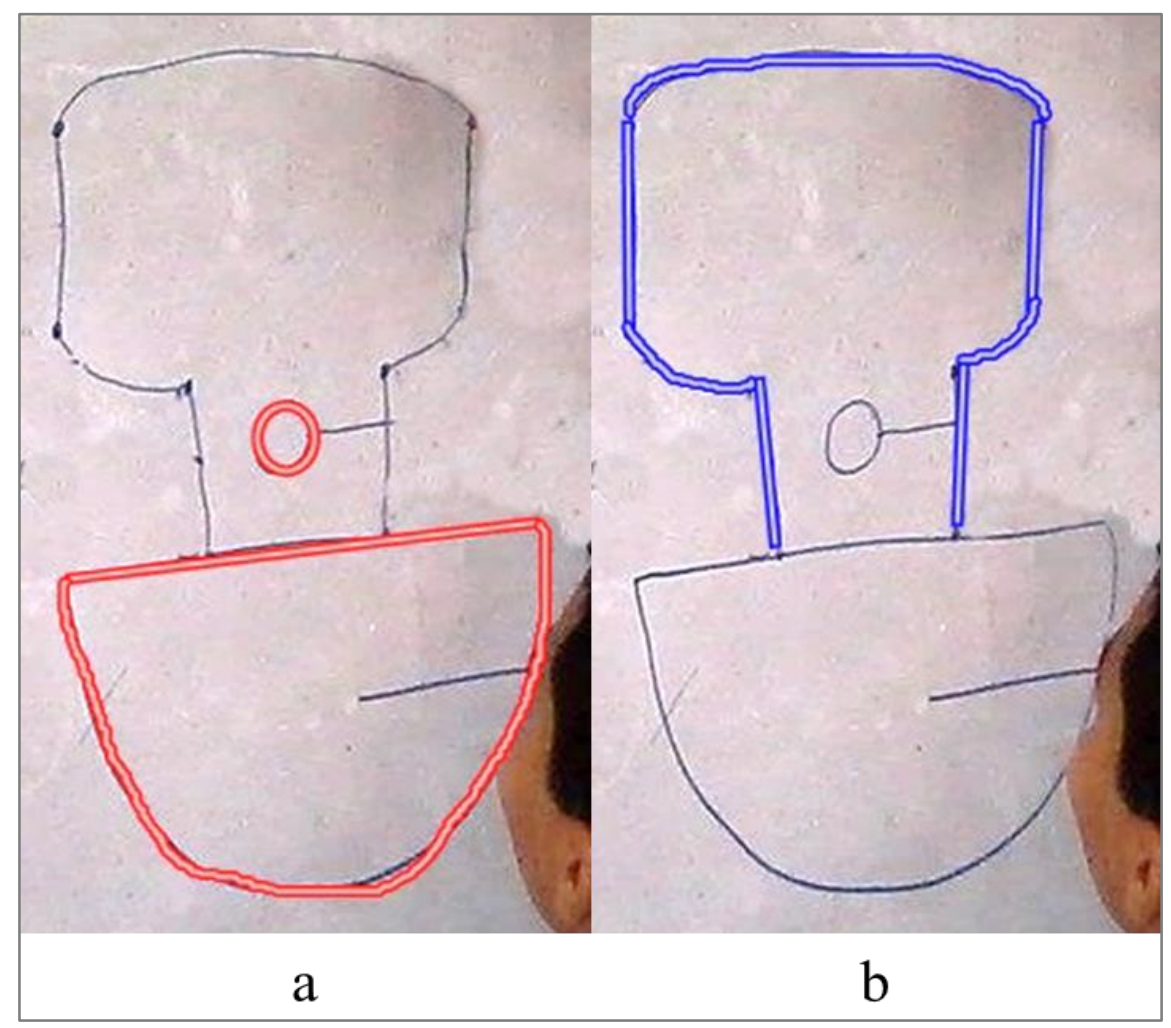

Fonte: Elaborado pelas autoras (2020)

Dos discursos transcritos, foi considerado o momento no qual Kevin e Rodrigo discutiram sobre o objeto geométrico que melhor podia representar a parte faltante no esboço do contrapeso. Nesse momento, Rodrigo discutia com Kevin a importância de realizar o esboço e identificar os objetos geométricos antes de fazer o desenho dinâmico, finalizando com a pergunta de qual seria o objeto que melhor representasse essa parte. Em seguida, Kevin responde que um quadrado, o que levou a Rodrigo a representar esse objeto no GeoGebra para verificar se, de fato, Kevin realmente conhecia as propriedades do quadrado.

Rodrigo Isso é muito importante, Kevin, porque às vezes você pode se perguntar: por : que não vamos aqui [referindo-se ao GeoGebra] e fazemos as coisas? Não, porque é sempre bom ver quais figuras vou construir lá. Se não o identifico, 
não construo nada lá. Que parte do esboço está faltando para identificar um objeto geométrico? Aquele ali? [enquanto Kevin aponta para a parte do esboço (Imagem 1)]

Imagem 1: Kevin apontando à parte que falta por identificar

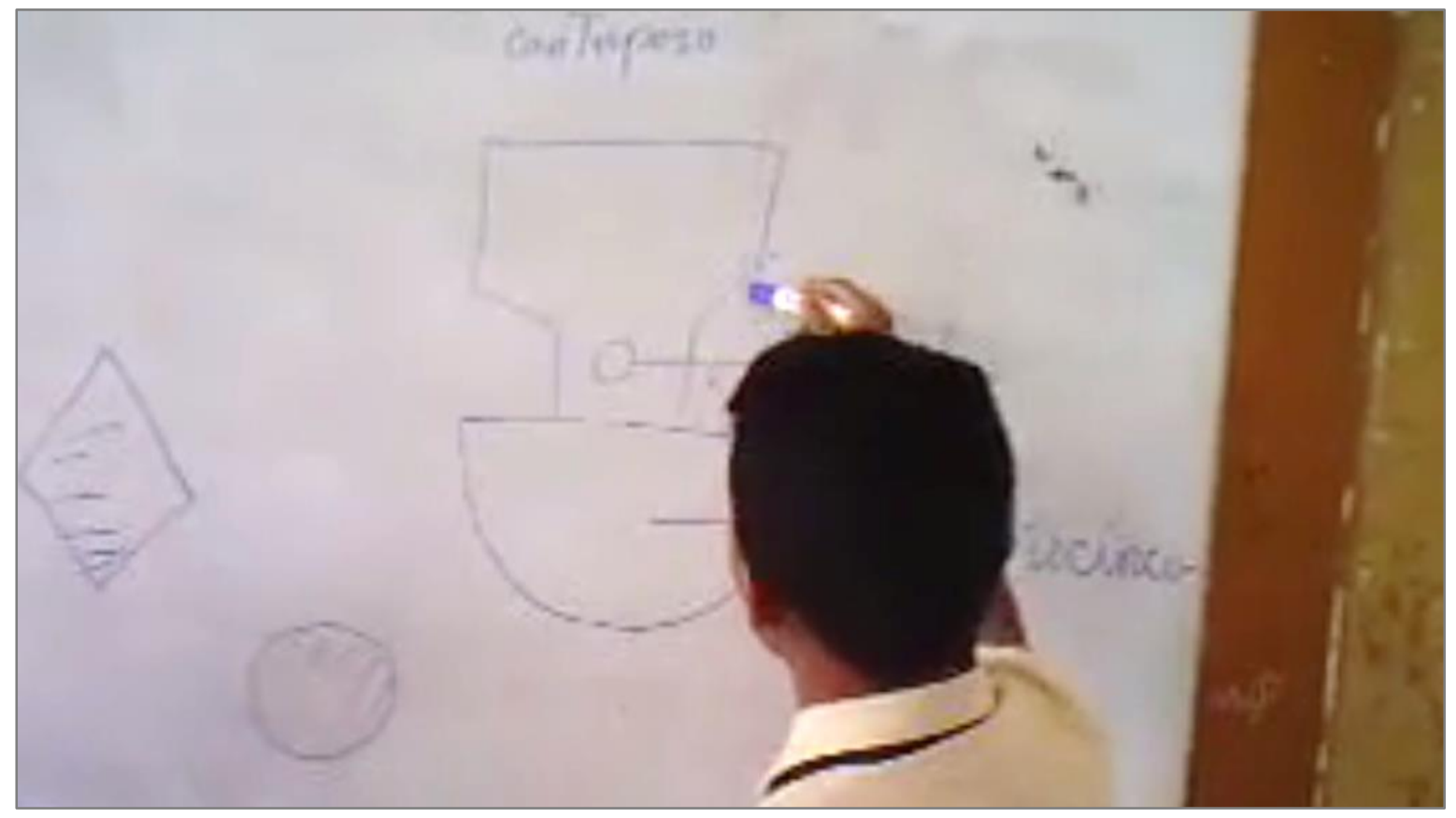

Fonte: Elaborado pelas autoras (2020)

Kevin: $\quad$ Parece mais um quadrado.

Rodrigo: Parece um quadrado, vamos ver. Você sabe o que é um quadrado? Porque eu lhe digo uma coisa, você me diz "para mim que é um quadrado", então para saber se é um quadrado ou não é a melhor figura, há que saber que é um quadrado. Por exemplo, lembra quando vocês me disseram "não ..." que você medisse "isso é um círculo", eu acho que era, e a Miyu disse "não, é uma circunferência". O que eu fiz? Eu fui para o conceito, certo? Eu fui aqui [apontando para o computador], o desenhei e tudo mais, certo?

Kevin: Certo!

Rodrigo: Nós vamos fazer o mesmo aqui [apontando no esboço ao suposto quadrado (Imagem 2)]. Não vamos discutir o que é um círculo, porque já sabemos o que é. Vamos ver se o quadrado é a melhor figura que essa parte daqui pode representar. O que você acha?

\section{Imagem 2: Rodrigo apontando ao suposto quadrado}




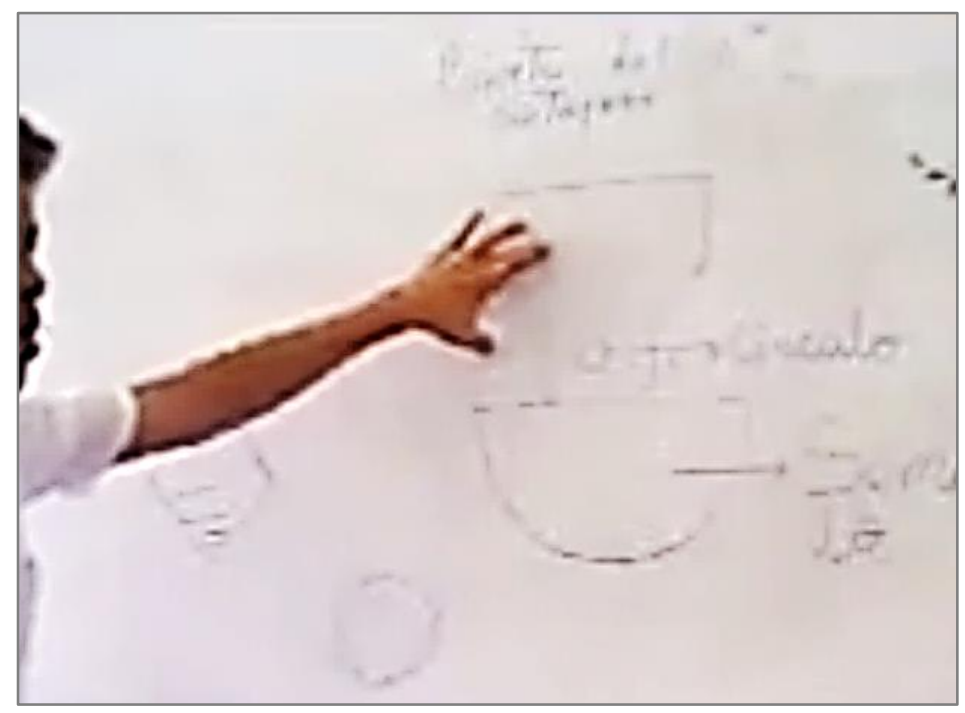

Fonte: Elaborado pelas autoras (2020)

Kevin: [confirma que sim com a cabeça]

Nesse momento, Rodrigo comenta com Kevin que o quadrado é um polígono especificamente o único quadrilátero regular, destacando que existem muitos tipos de quadriláteros que podem ser estudados depois, já que o interesse nesse momento era sobre o quadrado. Em seguida, Kevin comunica que o quadrado tem 4 lados e Rodrigo o convida a voltar a ver o esboço para ver se, de fato, a figura de aí tinha 4 lados. O aluno responde que só vê 3 .

Rodrigo: 3. O quadrilátero possui 4. Existem muitos tipos de quadriláteros, muitos. De repente, nas sessões que faremos mais tarde, podemos estudá-las. De todos esses tipos de quadriláteros, existe um chamado quadrado. $O$ seja, o

Kevin: 4. quadrado tem, então, quantos lados?

Rodrigo: Tem 4, já aí você tem que ir olhando, no esboço, sim tem 4 lados, viu? Ah?

Kevin: Só há 3.

Nesse discurso já se percebe que o aluno começa a duvidar sobre a figura que ele pensava que melhor pode representar essa parte do contrapeso. Rodrigo continua falando sobre o quadrado e suas características particulares, convidando a Kevin a verificar, no GeoGebra, se de verdade essas são as características. Depois indica para ele com qual ferramenta pode-se construir esse quadrado e começam a discutir sobre a quantidade de vértices que tem essa figura porque é o requerimento da ferramenta para poder construir o polígono regular. 
Rodrigo: Você vê 3? Bem, [...] Mas agora vamos prestar atenção aqui. Olha, aí você tem que ver se o quadrado é a melhor figura. Agora, porque digo que os livros dizem que o quadrado é um polígono regular? Um polígono regular é aquele que tem a mesma medida e ângulos internos de igual medida. Vamos ver se é verdade. Aqui está uma ferramenta, esta caixa que está aqui [apontando para a interface do GeoGebra], é de polígonos. Abre o menu [a caixa de ferramentas], polígono regular [apontando para a interface do GeoGebra]. Essa ferramenta permite desenhar polígonos regulares, de quantos lados? Daqueles que você deseja, de três em diante. Clique na tela, clique em outro lugar na tela e ele solicita o número de vértices. Se quisermos

Kevin: $\quad 4 k$ desenhar um quadrado, quantos vértices acha que podemos desenhar?

Rodrigo: 4! [eles colocam 4 na janela emergente]. Aí está (Imagem 3). [...] o que são vértices, Kevin? São esses cantos aqui, os cantos. Mas é claro, eu digo os cantos para você fazer a relação, mas eles são chamados de vértices e são pontos. Olha, eles são só pontos.

\section{Imagem 3: Quadrado construído no GeoGebra}

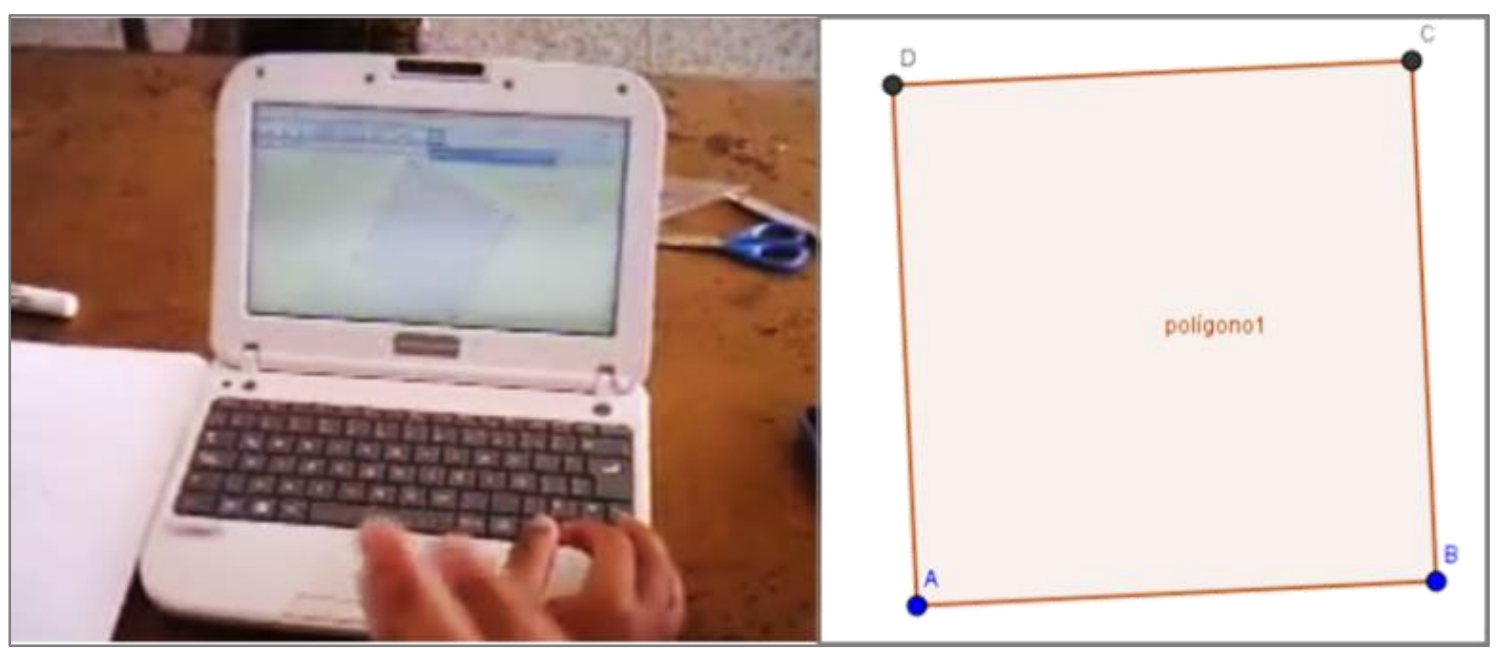

Fonte: Elaborado pelas autoras (2020)

Nesse momento percebe-se que começam a serem apresentadas para Kevin as características do quadrado por meio de sua representação no GeoGebra. Embora Rodrigo já tivesse as dito, talvez isso não seja suficiente para que o aluno faça desse conceito parte de seu conhecimento. Também se percebe que Kevin já estava refletindo sobre o dito pelo futuro professor que falou sobre os lados e ângulos, mas o aluno comunicou o número de vértices que deveria ter o quadrado, fato que evidencia uma reflexão de parte dele quanto à outra caraterística desta figura. Posteriormente, Rodrigo começou a refletir com Kevin sobre os lados do quadrado, convidando-o a medi-los. Considera-se que isso é realizado pelo futuro professor devido ao convite inicial ao aluno a verificar se o quadrado tinha as características que ele comentou. 
Rodrigo: Quais são os lados? Os segmentos que...

Kevin: Os segmentos...

Rodrigo: O que une quem?

Kevin: Aos vértices.

Rodrigo: Aos vértices, viu? Simples. Vamos medir os lados e eles têm que medir o mesmo

Kevin: Claro.

Rodrigo: Esta ferramenta aqui é a caixa de medidas. Se você abrir, aqui podemos medir as coisas. Podemos medir ângulos, podemos medir comprimentos ou distâncias, podemos medir área, muitas coisas. Vamos medir a distância [referindo-se à medida dos lados do quadrado desenhado na interface do GeoGebra] e você tocará nos lados, com um único clique, nos lados. Toque nos lados, no segmento [...] Novamente, selecione a ferramenta e toque no lado, clique no lado. Quanto mede?

Kevin: $\quad 3,49$.

Rodrigo: Meça o resto dos lados. [Silêncio enquanto mede um lado]. Meça o outro, meça o outro. Ok...

Kevin: $\quad$ Todos medem o mesmo [comprimento (Imagem 4)].

Imagem 4: Medição dos lados do quadrado

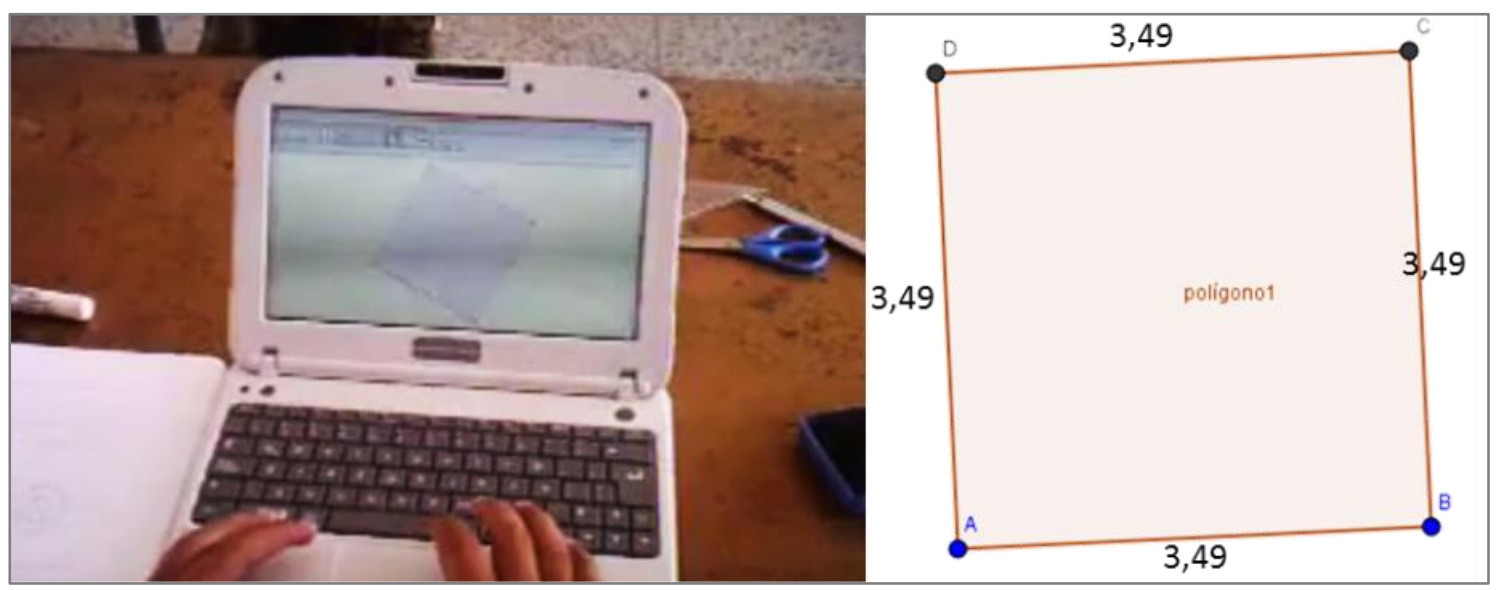

Fonte: Elaborado pelas autoras (2020)

No discurso de Kevin da para perceber que já ele estava fazendo, das caraterísticas do quadrado, parte de seu conhecimento ao comunicar o que estava visualizando no GeoGebra. Desse mesmo modo foi realizado para a medição dos ângulos.

Rodrigo: Todos medem o mesmo. Agora, o polígono regular de 4 lados diz que todos eles medem o mesmo, e os ângulos internos também medem o mesmo. Nós vamos medir os ângulos. Agora, qual ferramenta você vai selecionar? [enquanto assistem a tela].

Kevin: Ângulos!

Rodrigo: Ângulos! Eu acho que se você clicar dentro, aqui [apontando dentro do quadrado desenhado na interface do GeoGebra], ele fornece os ângulos. Continue! Viu? Quanto mede os ângulos?

Kevin: $\quad 90^{\circ}$.

Rodrigo: Apenas 1? Somente 2? Quantos medem $90^{\circ}$ ? 
Kevin: $\quad$ os 4! [(Imagem 5)].

Imagem 5: Medidas dos ângulos do quadrado

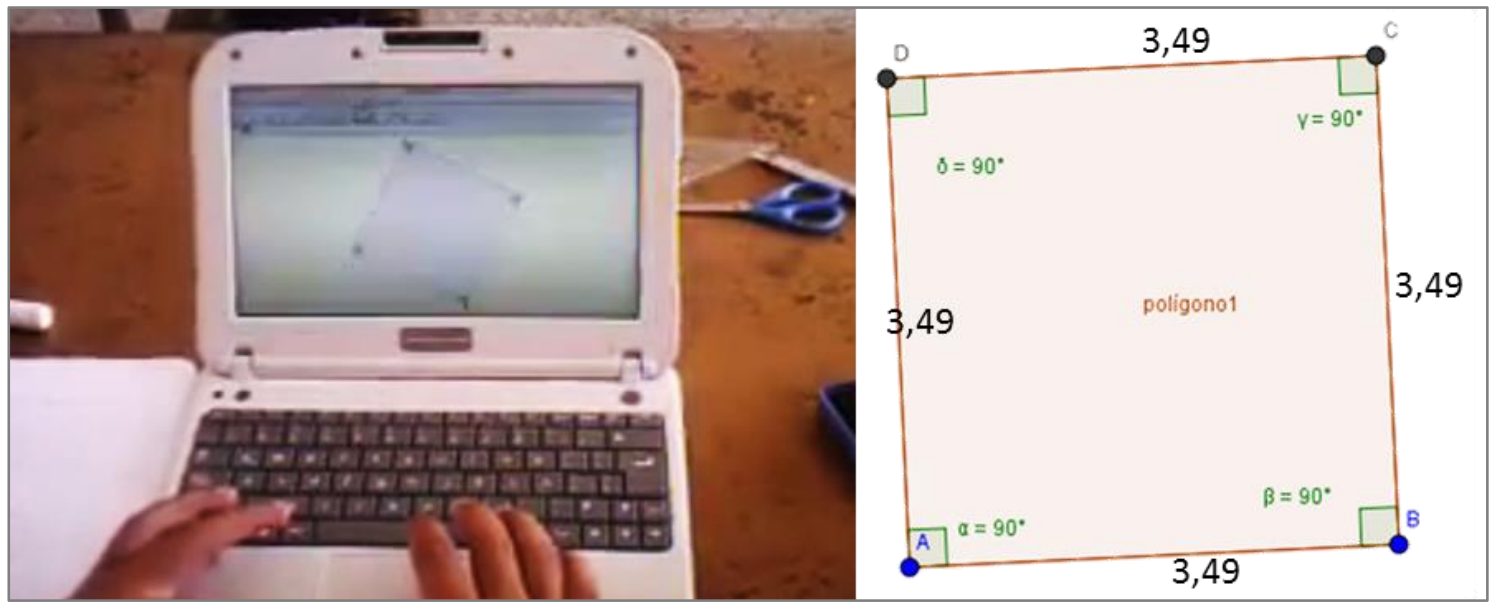

Fonte: Elaborado pelas autoras (2020)

Rodrigo: Os 4! Então, se todos os lados medem o mesmo e todos os ângulos medem o mesmo, que figura é essa?

Kevin: Um quadrado.

Neste discurso percebe as reflexões realizadas por Kevin, pois começa a comunicar qual ferramenta pode ser utilizada, a qual corresponde a um dos elementos que caracterizam ao quadrado, uma vez que estão verificando tais caraterísticas. Percebe-se que a representação possível no GeoGebra tem permitido aos participantes ir produzindo o conhecimento referido às ideias do que é um quadrado. Especificamente, isso é expresso por Kevin ao definir o que para ele é um quadrado, a partir do visualizado e experimentado ao construir e verificar as características desta figura com as ferramentas oferecidas pelo software, definição que é refinada por Rodrigo.

Rodrigo: Um quadrado! Isto é um quadrado. Então, como você pode-me definir um quadrado? Com base no que você está vendo, é um quadrado. Como você pode defini-lo?

Kevin: $\quad$ É uma figura geométrica composta de segmentos e tem a mesma medida... tem... os segmentos são iguais.

Rodrigo: Em medida! E que mais? Falta.

Kevin: $\quad$ E os ângulos também são iguais em medida.

Rodrigo: Exatamente! Olha, observe como não é difícil definir um objeto. O que você precisa ver são as características. Bem, podemos escrever isso mais tarde. Então, um quadrado, como você diz, é uma figura geométrica... Eu diria polígono, de uma vez, polígono. É um polígono que tem todos os lados, de igual medida, polígono de quatro lados. Porque pode haver polígonos de 5 lados, um polígono de 6 lados e um polígono de 30 lados. Um polígono de quatro lados e como são os lados? 
Kevin: Iguais!

Rodrigo: Em medida. E os ângulos?

Kevin: $\quad$ Os mesmos em medida.

Kevin, a partir do visualizado no GeoGebra enquanto experimentava para verificar as características do quadrado, conseguiu produzir seu conhecimento sobre o que é essa figura geométrica. Embora a comunicação não fosse totalmente direita, na fala há indícios de que ele refletiu sobre as questões que estavam sendo abordadas nesse momento. O relevante desta discussão é quando se percebe a transformação, a reorganização das ideias que Kevin tinha sobre o quadrado quando ele comunica que essa parte da qual estava faltando identificar a figura geométrica não era um quadrado.

Rodrigo: Perfeito! A questão é Kevin, isso [apontando para o quadrado na interface do GeoGebra] é parecido a isto que está aqui? [muda de posição e aponta para a parte do esboço que supostamente era um quadrado]

Kevin: Não!

Rodrigo: Então você tem certeza que isso é quadrado?

Kevin: Não é!

Rodrigo: Não é um quadrado.

Neste momento se percebe que a ideia inicial de Kevin sobre o que poderia ser um quadrado foi reorganizada por meio do visualizado e experimentado na representação do quadrado realizada no GeoGebra, o que pode ser considerado como parte da reflexão e transformação acontecida nele quanto a esse conceito. Além disso, percebe-se como a discussão coletiva dos envolvidos e a interação com o ambiente tecnológico ajudaram a ter a experiência de visualização e experimentação que permitiu continuar com a atividade de ESG. Na sequencia serão apresentadas as considerações finais sobre a situação.

\section{Considerações Finais}

No desenvolvimento deste trabalho foi realizada uma análise sobre a reorganização do conhecimento matemático a partir de experiências de visualização e experimentação na atividade de ESG, particularmente sobre a noção de quadrado. Especificamente, o intuito foi fornecer informações que possibilitem compreender como a visualização e experimentação acontecem na ESG, como isso pode ajudar aos envolvidos a reorganizar seus conhecimentos e como é percebido no desenvolvimento desta atividade mediada tecnologicamente. Como resultado desse processo, o aluno conseguiu transcender essas imprecisões que manifestou 
sobre o conceito do quadrado e sua relação com a parte do fenômeno que estava sendo simulado. Isso é mais relevante ainda porque, embora tenha passado por um sistema educacional no qual o quadrado seja estudado desde os anos iniciais, ele estava chegando quase a final dessa fase educativa apresentando fraquezas nas suas ideias sobre o que é um quadrado.

Desde a perspectiva teórica utilizada, considera-se que esses resultados se devem a três questões particulares: ao tipo de atividade que foi proposta, ao coletivo que esta realizando a atividade e ao meio tecnológico que foi utilizado. Com respeito à atividade, a mesma é gerada a partir das necessidades dos participantes em representar geometricamente uma parte de seu simulador. Quanto ao coletivo, na maioria, os sujeitos não são especialistas na Matemática, mas se mantem dispostos a produzir ou reorganizar seu conhecimento a partir do que está visualizando e/ou experimentando no GeoGebra. Sobre o meio tecnológico, o GeoGebra é um software de fácil manuseio para os participantes já que lhes oferece certas indicações de utilização das ferramentas, além de contar com diversos matérias escritos ou em vídeo apoiando o seu uso.

$\mathrm{Na}$ análise se percebeu que as questões de representar, refletir, transformar e comunicar estiveram presentes e apareciam de forma não linear. Porém, considera-se que faltou o assunto da generalização. Embora não tenha acontecido na experiência, vale destacar que a partir do que é visualizado e experimentado no GeoGebra é possível generalizar o conceito estudado. Só bastava movimentar ou arrastras o quadrado por algum de seus elementos livres para que Kevin visualizasse, enquanto experimentava, que essas propriedades do quadrado eram mantidas, ou seja, que qualquer quadrado tinha as mesmas características. Finalmente, no trabalho, pode-se observar uma relação entre a visualização, experimentação e reorganização do conhecimento matemático; no entanto, outros estudos são necessários para ajudar a desvendar ou caracterizar melhor esse tipo de relação.

\section{Agradecimentos}

Esta pesquisa foi realizada no contexto do projeto "A construção de animações e simulações no software GeoGebra e o Ensino e a Aprendizagem de Matemática”, aprovado e financiado pelo Conselho Nacional de Desenvolvimento Científico e Tecnológico (CNPqBrasil), de acordo com o resultado da chamada MCTIC/CNPq nº 28/20181. 


\section{Referências}

Bicudo, M. A. V. (2005). Pesquisa qualitativa: significados e a razão que a sustenta. Revista $\begin{array}{lll}\text { pesquisa qualitativa, } & \text { 7-26. }\end{array}$ https://scholar.google.com/scholar_url?url=http://rpq.revista.sepq.org.br/index.php/rpq/ article/view/7\&hl=es\&sa=T\&oi=gsb\&ct=res\&cd=0\&d=10686798123230863972\&ei=I RfUXqGcI6Sy9YP4omv8Aw\&scisig=AAGBfm3mK5OfQbRIIgcJ8UripxUQ1W8Tmg.

Bicudo, M. A. V. (2012). A pesquisa em educação matemática: a prevalência da abordagem qualitativa. Revista Brasileira de Ensino de Ciência e Tecnologia, 5(2), 15-26. Recuperado de: https://www.researchgate.net/profile/Maria_Bicudo/publication/270700726_A_pesquis a_em_educacao_matematica_a_prevalencia_da_abordagem_qualitativa/links/58206f2e 08ae12715afbba81/A-pesquisa-em-educacao-matematica-a-prevalencia-da-abordagemqualitativa.pdf.

Borba, M. C., \& Villarreal, M. E. (2005). Humans-with-media and the reorganization of mathematical thinking: Information and communication technologies, modeling, visualization and experimentation. Springer Science \& Business Media.

Hershkowitz, R., Ben-Chaim, D., Hoyles, C., Lappan, G., Mitchelmore, M., \& Vinner, S. (1990). Psychological aspects of learning geometry. Em Mathematics and cognition, 70-95. Cambridge University Press.

Laborde, C. (1997). Cabri Geómetra o una nueva relación con la geometría. En L. Puig (Ed.), Investigar y enseñar. Variedades de la educación matemática (pp. 33-48). Madrid: Una empresa docente.

Prieto, J.L. \& Gutiérrez, R.E. (Comps.). (2015). Memorias del I Encuentro de Clubes GeoGebra del Estado Zulia. Maracaibo, Venezuela: A.C. Aprender en Red. https://www.researchgate.net/publication/291043405_Memorias_del_I_Encuentro_de Clubes_GeoGebra_del_Estado_Zulia? sg=9AdWO2igkSuQYIsKZ675ISjpNisK7VA1P316bMVYhx6tGDWQsjpnDMrkbsWdDD_xAChoq4R49BfX1Ob5ez YazamkZFResu7p0pHUEnF.0dQFZULfbitBCgwn8Xy4z6TOaQhvYwUPXGFWcHG pKgT_pUEF15k97GoazXEvWbVdmxOjft6hQzA1GzbS8hH8EA.

Prieto, J.L. \& Gutiérrez, R.E. (Comps.). (2016). Memorias del II Encuentro de Clubes GeoGebra del Estado Zulia. Maracaibo, Venezuela: A.C. Aprender en Red. https://www.researchgate.net/publication/309206509_Memorias_del_II_Encuentro_de _Clubes_GeoGebra_del_Estado_Zulia?_sg=9AdWO2igkSuQYIsKZ675ISjpNisK7VA1P316bMVYhx6tGDWQsipnDMrkbsWdDD xAChoq4R49BfX1Ob5ez YazamkZFResu7p0pHUEnF.0dQFZULfbitBCgwn8Xy4z6TOaQhvYwUPXGFWcHG pKgT_pUEF15k97GoazXEvWbVdmxOjft6hQzA1GzbS8hH8EA.

Prieto, J.L. \& Gutiérrez, R.E. (Comps.). (2017). Memorias del III Encuentro de Clubes GeoGebra del Estado Zulia. Maracaibo, Venezuela: A.C. Aprender en Red. https://www.researchgate.net/publication/320538720_Memorias_del_III_Encuentro_de _Clubes_GeoGebra_del_Estado_Zulia?_sg=9AdWO2igkSuQYIsKZ675- 
ISjpNisK7VA1P316bMVYhx6tGDWQsjpnDMrkbsWdDD_xAChoq4R49BfX1Ob5ez YazamkZFResu7p0pHUEnF.0dQFZULfbitBCgwn8Xy4z6TOaQhvYwUPXGFWcHG pKgT_pUEF15k97GoazXEvWbVdmxOjft6hQzA1GzbS8hH8EA.

Rubio, L., Prieto, J.L. \& Ortiz, J. (2015). La matemática en la simulación con GeoGebra. Una experiencia con el movimiento en caída libre. International Journal of Educational Research and Innovation (UJERI), 2, 90-111. http://upo.es/revistas/index.php/IJERI/article/view/1586.

Torregrosa, G. (2002). Visualización y aprendizaje de la Geometría. Universidad de Alicante, España.

Tikhomirov, O.K. (1981). "The psychological consequences of computarization". Em J.V. Wertsch (ed.), The concept of activity in Soviet Psychology, Nueva York, M.E. Sharpe, pp. 256-278.

Villa, J. A., Vélez, L., Rojas, C. \& Borba, M. C., 2013. Visualización de conceptos matemáticos. GeoGebra en la reorganización de los modos de producción de conocimiento matemático. En López, L. (Ed), Una visión de las ciencias básicas. Modelación y aplicación a casos reales (pp, 65-80). Sello Editorial.

Villa, J. A., \& Ruiz, M. (2010). Pensamiento variacional: seres-humanos-con-GeoGebra en la visualización de noción variacional. Educação Matemática Pesquisa, 12(3), 514-528. http://bibliotecadigital.udea.edu.co/handle/10495/3921.

Villa, J. A, \& Borba, M. C. (2011). Humans-with-media en la producción del conocimiento matemático: el caso de Geogebra. 12 Encuentro Colombiano de Matemática Educativa. http://funes.uniandes.edu.co/1778/.

Villarreal, M. E. (2003). Pensamiento matemático, cálculo diferencial y computadoras. Educación matemática, 15(1), 99-122. http://funes.uniandes.edu.co/13031/.

Villarreal, M. E. (2004). Transformaciones que las tecnologías de la información y la comunicación traen para la educación matemática. Yupana, 1(1), 41-55. http://funes.uniandes.edu.co/16229/.

Villarreal, M. E. (2012). Tecnologías y educación matemática: necesidad de nuevos abordajes para la enseñanza. Virtualidad, Educación y Ciencia, 3(5), 73-94. https://revistas.psi.unc.edu.ar/index.php/vesc/article/view/3014.

Villarreal, M. E. (2013). Humanos-con-medios- un marco para comprender la producción matemática y repensar prácticas educativas. Em Miranda, E. \& Pauciulli, N. (Ed), Formación de profesores, curriculum, sujetos y prácticas educativas. La perspectiva de la investigación en Argentina y Brasil (pp, 85-122). Córdoba, Universidad de Córdoba.

Autoras

Stephanie Díaz-Urdaneta

Mestra em Educação em Ciência e Matemática pela Universidade Federal do Paraná (UFPR Brasil). Licenciada em Educação menção Matemática e Física pela Universidad del 
Zulia (LUZ - Venezuela). Secretária da Asociación Aprender en Red (Venezuela). Membro do Grupo de Pesquisa sobre Tecnologias na Educação Matemática (GPTEM Curitiba, Brasil). Temas de pesquisa: Formação de Professores e Uso de Tecnologias Digitais na Educação Matemática. E-mail: stephaniediazurdaneta@gmail.com

Luzia Regis Narok Pereira

Mestra em Educação em Ciência e Matemática pela Universidade Federal do Paraná (UFPR Brasil). Especialista em Metodologia do Ensino da Matemática pela Universidade Leonardo Da Vinci (Uniasselvi - Brasil). Licenciada em Matemática pela Uniasselvi. Professora de Matemática e Programação da rede estadual do Paraná. Temas de pesquisa: Uso de Tecnologias Digitais na Educação Matemática e Formação de Professores. E-mail: matematicaluzia@gmail.com 